\title{
Incidência e Caracterização dos Casos de Sífilis Congênita na Maternidade de um Hospital do Sudoeste Baiano
}

\author{
Débora Maria Santos Azevedo ${ }^{1}$; Rosilaine Barbosa Silva Reis ${ }^{2}$; Mauro Fernandes Teles ${ }^{3}$
}

\begin{abstract}
Resumo: Dentre as diversas doenças que podem ser transmitidas durante a gestação, a sífilis aponta as maiores taxas de transmissão. Embora seja uma doença com tratamento simples e de fácil acesso, ainda segue como um grave problema de saúde pública. A sífilis congênita advém da gestante infectada resultando na disseminação hematogênica do Treponema pallidum por meio da via placentária para o feto. É classificada como doença de notificação compulsória para fins de vigilância epidemiológica, devendo ser notificada em uma ficha de notificação e/ou investigação. O objetivo deste estudo foi determinar a incidência e caracterização dos casos de sífilis congênita, na maternidade de um hospital do sudoeste baiano. Trata-se de uma pesquisa descritiva transversal em que a população do estudo compreendeu todos os casos investigados e notificados no ano de 2017. O principal instrumento para coleta de dados foi a ficha de notificação/investigação de sífilis congênita do Sistema de Informação de Agravos de Notificação (SINAN), onde foram retiradas todas informações dos casos de sífilis congênita. Foram notificados um total de 60 casos de sífilis congênita, com incidência estimada de 1,35 casos por mil nascidos vivos. Os resultados demonstraram associação com a variável cor materna parda $(67,3 \%)$. Foi observado deficiência quanto ao preenchimento da ficha de notificação, levando a um número significativo de dados faltantes, o que corrobora a necessidade de desenvolver estratégias para aprimorar a qualidade dos sistemas de saúde. Os dados evidenciaram ainda, existir desafios para o alcance da erradicação da sífilis congênita, uma vez que é crescente o número de casos no país.
\end{abstract}

Palavras-chave: Sífilis Congênita; Ficha de notificação; Incidência.

\section{Incidence and Characterization of Cases of Congenital Syphilis in a Maternity Hospital of Southwest}

\begin{abstract}
Among the various diseases that can be transmitted during pregnancy, syphilis shows the highest rates of transmission. Although it is a disease with simple treatment and easy access, it still follows as a serious public health problem. Congenital syphilis comes from the infected pregnant woman resulting in hematogenous dissemination of Treponema pallidum via the placental route to the fetus. It is classified as notifiable disease for epidemiological surveillance purposes and must be notified in a notification and / or investigation form. The objective of this study was to determine the incidence and characterization of congenital syphilis cases in the maternity ward of a hospital in southwest Bahia. It is a cross-sectional descriptive study in which the study population comprised all cases investigated and reported in the year 2017. The main instrument for data collection was the notification / investigation form of congenital syphilis of the Notification Disease Information System (SINAN), where all information on the cases of congenital syphilis was withdrawn. A total of 60 cases of congenital syphilis were reported, with an estimated incidence of 1.35 cases per thousand live births. The results showed an association with the variable maternal brown color $(67.3 \%)$. A deficiency was observed in filling out the notification form, leading to a significant number of missing data, which corroborates the need to develop strategies to improve the quality of health systems. The data also showed that there are challenges to the eradication of congenital syphilis, since the number of cases in the country is increasing.
\end{abstract}

Keywords: Congenital Syphilis; Notification form; Incidence.

\footnotetext{
${ }^{1}$ Acadêmica em Farmácia Generalista pela Faculdade Independente do Nordeste - FAINOR. azeveddodebora@gmail.com

${ }^{2}$ Nutricionista, Esp. Preceptora do Curso de Farmácia na Faculdade Independente do Nordeste - FAINOR. Vitória da Conquista-Bahia. rosilaine.bsilva@ hotmail.com

${ }^{3}$ Farmacêutico, MSc. Docente do Curso de Farmácia na Faculdade Independente do Nordeste - FAINOR. Vitória da Conquista-Bahia.mauro@fainor.com.br
} 


\section{Introdução}

A sífilis é uma patologia que vem se estendendo por centenas de anos na humanidade, mesmo apresentando um tratamento simples e acessível, ainda segue como um sério problema de saúde pública. Trata-se de uma infecção causada pelo Treponema pallidum de evolução crônica e muitas vezes assintomática e embora seja uma doença de transmissão sexual pode ser transmitida também por via direta da placenta da mãe para o feto ou por meio do canal do parto, caracterizando a sífilis congênita (SILVA; et al., 2017; LAFETÁ; et al., 2016).

Aproximadamente dois milhões de gestantes são infectadas pela sífilis a cada ano, sendo que a maior parte delas não efetua o teste para sífilis e quando realizam não são tratadas corretamente ou nem mesmo recebem tratamento. A sífilis aponta as maiores taxas de transmissão vertical no meio as diversas doenças que podem ser transmitidas no decorrer da gestação (NONATO; MELO; GUIMARÃES, 2015; REIS et al., 2018).

A sífilis congênita advém da gestante infectada resultando na disseminação hematogênica do Treponema pallidum por meio da via placentária para o feto. Apresenta para efeito de classificação, duas fases: quando o diagnostico ocorre até dois anos de vida identificada como precoce, e após esse período classificada como tardia (BRASIL, 2016).

O aumento dos casos de sífilis congênita apresenta como condição principal a assistência pré-natal inadequada. Diferentes estudos relacionam a doença com a baixa renda, infecção pelo HIV, uso de drogas e acesso ao sistema de saúde. As condições de risco especificas compreendem gravidez na adolescência, raça/cor, nível de escolaridade inferior e sobretudo a assistência pré-natal (NONATO; MELO; GUIMARÃES, 2015).

A sífilis congênita é classificada como doença de notificação compulsória desde 1986, para fins de vigilância epidemiológica. Os agravos de notificação compulsória devem ser notificados em uma ficha de notificação e/ou investigação, essa ficha é preenchida pelas unidades assistências para cada paciente e depois repassada para serviços encarregados pela informação e/ou vigilância epidemiológica das Secretarias de Saúde (GUIMARAES; et al., 2018; SISTEMA NACIONAL DE AGRAVOS DE NOTIFICAÇÃO - SINAN, 2018).

Segundo dados do boletim epidemiológico de sífilis (2016), foram notificados um total de 136.999 casos de sífilis congênita no Brasil entre o ano de 2000 a 2016, com maior prevalência nas regiões sudeste e nordeste. Em 2011 o número total de casos notificados sífilis congênita em menores de um ano de idade no Brasil foi de 9.490. Em 2012 houve 11.232 notificações de casos em crianças com idade igual a menor que sete dias, sendo que o percentual maior segundo faixa etária da mãe está entre 20 a 29 anos correspondendo um total 52,4\% 
(BRASIL, 2016). Neste contexto, esse estudo teve o objetivo de determinar a incidência e caracterização dos casos de sífilis congênita na maternidade de um hospital do sudoeste baiano no ano de 2017.

\section{Metodologia}

Trata-se de uma pesquisa descritiva transversal. A população do estudo compreendeu todos casos de sífilis congênita investigados e notificados, incluindo abortos e natimortos, realizado na maternidade de um hospital de um município do sudoeste baiano notificados no ano de 2017.

O presente estudo teve como base dados secundários, onde a principal fonte de informação utilizada foi a ficha de notificação/investigação da sífilis congênita do Sistema de Informação de Agravos de Notificação - SINAN, utilizada como acesso do número total e todas informações dos casos de sífilis congênita notificados no período e maternidade da região estudada. É considerada uma ferramenta de suma importância para a organização das ações programáticas de prevenção e assistência deste agravo.

O hospital designado para pesquisa dispõe de vigilância epidemiológica na detecção e investigação de doenças de notificação compulsória. É considerado o hospital com maior quantidade de partos realizados no município, referência em todo estado da Bahia em atendimento materno-infantil. O hospital oferece Centro Obstétrico com pré-parto e parto; UTI Neonatal com dez leitos, semi-intensiva com 15 leitos. Sendo que o hospital realiza por mês, em torno de 350 partos.

A coleta de dados foi feita a partir da ficha de notificação/investigação da sífilis congênita referentes ao ano de 2017. Como definição da quantidade de pacientes a serem analisados, levou-se em consideração a população total atendida no ano. A partir da avaliação da ficha de notificação, analisou-se os métodos laboratoriais como os testes treponêmicos e não-treponêmicos, período de realização do diagnostico, tratamento medicamentoso prescrito e encaminhamento dos recém-nascidos com sífilis congênita.

Foram consideradas para o estudo as variáveis maternas como idade, cor da pele, escolaridade, realização do pré-natal, diagnóstico e tratamento. Foi considerado também a variável diagnostico laboratorial e tratamento do recém-nascido. Cada uma dessas variáveis contidas na ficha de notificação/investigação da sífilis congênita do SINAN. 
Como definição da variável tratamento, a forma adequada seria o uso correto da Penicilina G cristalina na dose de $50.000 \mathrm{UI} / \mathrm{Kg}$ por via endovenosa, a cada 12 horas (nos primeiros 7 dias de vida) durante 10 dias, ou uso da Penicilina G benzatina na dose única de $50.000 \mathrm{UI} / \mathrm{Kg}$. Na variável diagnóstico, foi considerado como correto pacientes que realizaram os testes treponêmico e não treponêmico. Considerado como incorreto recém-nascidos que não realizaram o teste treponêmico anticorpo total, no caso das mães quando a realização de apenas um teste laboratorial ou quando o resultado foi ignorado/sem resultado na ficha de notificação.

Com os dados obtidos nos questionários, foi criado um banco de dados em planilha Excel (versão 2010, Microsoft®) e, posteriormente, exportado para o programa estatístico EpiInfo (versão 7.1.5.2).

O estudo foi submetido e aprovado pelo Comitê de Ética e Pesquisa da Fundação Pública de Saúde de Vitoria da Conquista com parecer número 2.867.083, segundo os padrões exigidos pela Resolução No 466/2012.

\section{Resultados}

Verificou-se que do total de partos ocorridos na maternidade do hospital no ano de 2017, foram notificados 60 casos de sífilis congênita, com incidência estimada de 1,35 casos/1.000 nascidos vivos.

Das 60 mães estudadas, 57 tinham idade definida e 3 não constavam informações na ficha de notificação/investigação do SINAN. A idade média materna foi de 25 anos, com faixa etária de 22 a 30 anos. Uma maior prevalência de sífilis na gestação foi observada em mulheres de cor parda (67,3\%), houve presença constante de dados em branco, em que 11 pacientes não continham informações na ficha.

Quanto a escolaridade das mães, relatou mais que 8 anos de estudo e 12 encontradas como respostas vazias ou sem preenchimento na ficha.

Das gestantes com diagnóstico positivo de sífilis, $90 \%$ fizeram o acompanhamento do pré-natal, apenas duas não realizaram e cinco gestantes pelo qual apresentou-se como em branco. A unidade de saúde para realização das consultas da maioria dos casos foi em Vitoria da Conquista (50\%), o mais ocorreram em cidades circunvizinhas.

$\mathrm{Na}$ Tabela 1, é possível observar descrição das características maternas, como perfil sociodemográfico, escolaridade e realização do pré-natal das mães. 
Tabela 1. Análise descritiva do perfil-materno dos casos de sífilis congênita.

\begin{tabular}{lcclrr}
\hline VARIÁ VEIS & $\mathbf{N}$ & $\mathbf{( \% )}$ & VARIÁVEIS & $\mathbf{N}$ & $\mathbf{( \% )}$ \\
\hline Idade & & & Cor & \\
$14-21$ & 16 & 28,1 & Branca & 12 & 23,1 \\
$22-30$ & 27 & 47,4 & Preta & 1 & 1,9 \\
$31-40$ & 14 & 24,6 & Parda & 35 & 67,3 \\
Total & 57 & 100 & Ignorado & 4 & 7,7 \\
Escolaridade & & & Realizou Pré-natal & & \\
Sem instrução & 2 & 5 & Sim & 50 & 90,9 \\
1-5 anos de estudo & 6 & 15 & Não & 2 & 3,6 \\
6-10 anos de estudo & 23 & 57,5 & Ignorado & 3 & 5,5 \\
>=11 anos de estudo & 9 & 22,5 & Total & 55 & 100 \\
\hline
\end{tabular}

Fonte: Dados da pesquisa.

No que se refere ao diagnóstico laboratorial de sífilis das mães e recém-nascidos, quarenta e sete mulheres $(78,3 \%)$ receberam o diagnóstico correto. Dos recém-nascidos, apenas 28,3\% não receberam o diagnóstico de forma correta (Tabela 2). Foi considerado como diagnóstico correto os RN que realizaram o VDRL permitindo assim correlacionar com o da mãe e considerado incorreto aqueles pacientes que realizaram o teste treponêmico anticorpo total e/ou que não apresentou resultado ou não tinha informação na ficha de realização do exame. Apenas seis pacientes apresentaram títulos de VDRL no RN maior que o da mãe o que confirma laboratorialmente sífilis congênita.

Entre as mães diagnosticadas, quarenta e três foram tratadas, porém somente trinta e três $(63,5 \%)$ receberam o tratamento adequado, quatro não realizaram, cinco delas considerado como ignorado e oito sem informações na ficha. Já no que se refere ao tratamento dos recémnascidos, foi considerado como correto todo os casos (95\%), que haviam prescrição de tratamento do uso da Penicilina, apenas dois considerados como não tratados e os demais como vazios e/ou em branco (Tabela 2). O cálculo da dosagem do tratamento é baseado no peso dos recém-nascidos. 
Tabela 2. Análise descritiva das mães e recém-nascidos segundo diagnóstico laboratorial e tratamento.

\begin{tabular}{lcclcc}
\hline VARIÁVEIS & $\mathbf{N}$ & $\mathbf{( \% )}$ & VARIÁVEIS & $\mathbf{N}$ & $\mathbf{( \% )}$ \\
\hline Diagnóstico (mãe) & & & Diagnóstico (recém-nascido) & & \\
Correto & 43 & 71,7 & Correto & 43 & 71,7 \\
Incorreto & 17 & 28,3 & Incorreto & 17 & 28,3 \\
Total & 60 & 100 & Total & 60 & 100 \\
Tratamento & & & Tratamento & & \\
Adequado & 33 & 63,5 & Adequado & 38 & 95 \\
Inadequado & 10 & 19,2 & Inadequado & 0 & 0 \\
Não realizado & 4 & 7,7 & Não realizado & 2 & 5 \\
Ignorado & 5 & 9,6 & Ignorado & 0 & 0 \\
Total & 52 & 100 & Total & 40 & 100 \\
\hline
\end{tabular}

Fonte: Dados da pesquisa.

Dos casos de sífilis congênita houve sete casos de natimorto. Apenas seis recémnascidos foram considerados como sintomáticos, entretanto não apresentava informações sobre os sinais e sintomas. Não foi encontrado nenhuma alteração do teste treponêmico no Líquor, sendo que em alguns casos não foi realizado o exame.

\section{Discussão}

Os resultados aqui mostrados, discutem em sua primeira parte, a taxa de incidência de casos de sífilis congênita na maternidade do hospital estudado. A incidência estimada foi de 1,35 casos por mil nascidos vivos no hospital, no ano de 2017. De acordo o Boletim Epidemiológico de Sífilis do Ministério da Saúde, a taxa de incidência de sífilis congênita deve ser $\leq$ 0,5 caso/1.000 nascidos vivos para a competência dos municípios ao seguimento de certificação nacional (BRASIL, 2017).

Dados brasileiros atuais, referidos no Boletim Epidemiológico de Sífilis de 2016, apresentam não só o insucesso em alcançar essa meta, como também progressivas taxas de incidência e mortalidade infantil por essa doença. A taxa de incidência de sífilis congênita no Brasil em menores de um ano passou de 1,7 casos/1.000 nascidos vivos em 2004 para 6,8 
casos/1.000 nascidos vivos em 2016. O Nordeste se encontra como a segunda região com maior taxa (7,0 casos/1.000 nascidos vivos), acima da taxa nacional (BRASIL, 2017).

Os dados apontaram que do perfil das mães notificadas com sífilis, a média de idade foi de 25 anos com faixa etária de 22 a 30 anos. Essa faixa etária de mulheres adultas jovens coincide com um outro estudo realizado no Brasil nesse mesmo período, por Barcellos et al., 2018, que também determinou faixa etária aproximada. Apesar da idade das mulheres infectadas retratar a prática da atividade sexual precoce, principalmente no que se refere a adolescentes, é importante a prevenção da população em geral (LAFETÁ et al., 2016).

Segundo Domingues et al., 2014, mulheres de cor preta ou parda, apresentam coberturas menores de testagem e são as que tiveram maior predominância de sífilis na gestação, alertando para oportunidades perdidas de diagnóstico e maior risco de transmissão vertical. Neste estudo foram encontrados resultados semelhantes no que se refere a características como raça/cor, demonstrando que mães de cor parda $(67,3 \%)$, apresentaram prevalência maior quando comparada a raça/cor branca.

De acordo a escolaridade, aspecto relevante para caracterização socioeconômica das mães estudadas, verificou-se um número elevado de 57,5\% (n=23), com escolaridade superior a 8 anos, representando um perfil diferenciado e identificando um bom grau de escolaridade, o que acaba se tornando preocupante pois espera que essa população tenha um nível de conhecimento melhor acerca da doença. Diferentemente de outros estudos como o de Chaves et al., (2014), e Domingues et al., (2014), que demonstraram resultados de maior prevalência de sífilis na gestação em adolescentes ou jovens com baixa escolaridade, indicando maior vulnerabilidade social.

Com relação a adesão ao pré-natal, observou-se de forma positiva que 90,9\% ( $\mathrm{n}=50)$, realizaram as consultas. Foi no momento do pré-natal que as gestantes obtiveram o diagnóstico da sífilis congênita, seguido pelo momento do parto. Moreira et al., 2017, também apresenta resultado parecido em seu estudo, em que o pré-natal foi também o momento de maior notificação dos casos. Enfatiza para uma assistência pré-natal de qualidade com diagnóstico precoce de forma a garantir a minimização do tempo de exposição ao treponema.

Quanto ao diagnóstico laboratorial das mães, segundo o Ministério da Saúde (MS), deve-se proceder dois testes, um treponêmico e outro não treponêmico para confirmação, se os dois testes apresentarem resultados discrepantes é necessário a efetuação de um outro treponêmico de metodologia diferente da usada inicial (BRASIL, 2006). Resultados mostraram que 43 mães receberam o diagnóstico correto, de acordo o que é preconizado pelo MS. As com diagnostico incorreto $(28,3 \%)$, foram assim consideradas quando realizado apenas um teste 
laboratorial, não foi realizado o terceiro teste laboratorial quando necessário ou quando o resultado foi ignorado/sem resultado na ficha.

Já em relação ao diagnóstico dos recém-nascidos, 71,7\% tiveram o diagnóstico correto. Pensando no diagnóstico laboratorial do bebê, o mesmo pode ser realizado por pesquisa direta, com identificação do agente etiológico ou testes sorológicos. O Ministério da Saúde preconiza para os casos em que a mãe não foi tratada ou tratadas de forma inadequada, independente do resultado do VDRL do RN, realizar hemograma, radiografia de ossos longos e outros exames quando indicados. Quando as mães adequadamente tratadas, realizar o VDRL em amostra de sangue periférico, se este for reagente com títulos maior que o da mãe ou apresentando alterações clínicas, realizar os mesmos exames já citados anteriormente e a análise do LCR (BRASIL, 2006).

No que diz respeito à realização do tratamento das mães, verificou-se no estudo França et al., 2014, de forma semelhante a este estudo, casos notificados de sífilis que apresentaram tratamento inadequado, sendo este caracterizado como qualquer tratamento que não seja com a penicilina, ou que esteja incompleto ou incompatível com a fase da doença. Devendo ser realizado de acordo com MS, o uso da penicilina 30 dias antes do parto, considerando o estágio da doença.

Para o tratamento da sífilis congênita, o MS preconiza o uso da Penicilina G cristalina na dose $50.000 \mathrm{UI} / \mathrm{Kg}$ ou Penicilina G procaína dose única nos casos em que as mães não foram tratadas ou tratadas de forma inadequada, já em casos em que a mãe foi tratada adequadamente efetuar o tratamento com Penicilina G cristalina. Se for assintomático e o VDRL não for reagente, proceder apenas com seguimento clínico-laboratorial, quando na impossibilidade do seguimento deve-se fazer o tratamento com penicilina $\mathrm{G}$ benzatina na dose única de 50.000 $\mathrm{UI} / \mathrm{Kg}$ (BRASIL, 2017). No presente estudo, como as informações ficaram vagas no que se refere também ao peso dos $\mathrm{RN}$, impossibilitou avaliar se realmente realizado de forma adequada o tratamento. Evidencia-se que em $95 \%$ haviam informações de prescrição, os outros $5 \%$ dos casos não continham informações sobre o tratamento, dado que corrobora com o estudo de Saracenitiseis et al., 2016.2018.

Foi verificado no estudo a presença de dados ignorados, em brancos, ausência de informações ou variáveis de grande relevância não preenchidas na ficha de notificação/investigação. Demonstrando assim uma deficiência quanto ao preenchimento da ficha de notificação dos casos de sífilis de congênita. Isso possibilita compreender sobre a forma como profissionais de saúde procedem com preenchimento de instrumentos de notificação, 
visto que apenas avaliar os dados não são suficientes, é necessário um acompanhamento constante dessas bases de dados.

Este estudo foi semelhante ao de Saraceni et al., 2015, que ressalta ainda existir lacunas para melhor qualificação da informação que é enviada para a base de dados oficial do SINAN.

As condições relacionadas à sífilis congênita encontradas no estudo, apontam falhas na assistência e necessidade de garantir novas estratégias com o propósito de reduzir a transmissão vertical da sífilis, entre elas uma melhor capacitação dos profissionais de saúde como a própria vigilância epidemiológica quanto ao preenchimento da ficha. Segundo Silva et al., 2014, durante a formação acadêmica dos profissionais da área da saúde é importante considerar a maneira como a informação e o conhecimento acerca das doenças de notificação compulsória seja transmitida, pensando que essa responsabilidade pela identificação e investigação destas doenças não seja uma atribuição exclusiva do médico e sim de todos profissionais.

A ficha de notificação/investigação de sífilis congênita do SINAN, é vista como um dos sistemas de informação fundamental para o meio de trabalho da vigilância epidemiológica, como uma das medidas de controle, pois possibilita coletar e divulgar dados sobre o agravo de notificação compulsória, possibilitando que seja analisado o impacto da doença (ARAÚJO; SILVA, 2013). É um sistema que está em desenvolvimento continuo, pois, a demanda cada dia é mais complexa, ressaltando inclusive há necessidade de atualização da ficha de notificação, pois, apresenta informações não mais necessárias como o tratamento do parceiro sexual, que de acordo o Ministério da Saúde, não sendo mais considerada essa informação (BRASIL, 2017).

\section{Conclusões}

Esse estudo, trouxe resultados sobre a incidência e caracterização dos casos notificados de sífilis congênita na maternidade do hospital estudado. Aponta para a necessidade de alguns ajustes e indicando ainda a situação atual da doença no país. Os achados revelaram, que apesar de metas governamentais para erradicação da doença, ainda é crescente o número de casos no Brasil nos últimos anos.

Como uma das estratégias para redução da incidência de casos de sífilis congênita, deve ser considerado importante a forma como os profissionais de saúde devem proceder, a começar pelo o enfoque das gestantes na realização do pré-natal, diagnóstico, tratamento e de extrema importância o preenchimento da ficha de notificação/investigação do SINAN. 
Os dados encontrados indicam a necessidade de melhor qualificação do preenchimento da ficha de notificação/investigação da sífilis congênita, verificou-se uma situação desfavorável quanto ao preenchimento das variáveis em geral, uma vez que em muitos casos os dados referentes as variáveis não foram informadas, o que dificultou melhores análises. Sugere-se assim melhor qualificação das informações notificadas, consideradas de grande relevância para a base de dados do SINAN.

Mesmo que evidentes falhas dos serviços de saúde, não se pode desconsiderar a relevância das condições de vida relacionada a doença. Os resultados apresentaram relevância de variável sobre condições de vida, uma vez que foi observado associação com a variável cor materna, em que as mães com maior predominância de sífilis na gestação foram de cor parda, alertando para oportunidades perdidas de diagnóstico e maior risco de transmissão vertical. É importante que o planejamento em saúde leve em consideração todos essas questões, para que a resposta ao problema seja precisa.

\section{Referências}

ARAÚJO, M. M. P.; SILVA, C. G. A importância do sistema de informação de agravos de notificação - SINAN para a vigilância epidemiológica do Piauí. Revista Interdisciplinar Ciências e Saúde, ISSN, 2358-6966, 2013.

BRASIL, Protocolo Clínico e Diretrizes Terapêuticas para prevenção da Transmissão Vertical e HIV, Sífilis e Hepatites Virais. Ministério da saúde. Brasília - DF 2015. Disponível em: $<$ http://www.sgvs.saude.ms.gov.br/wpcontent/uploads/sites/101/2017/05/pcdt_transmissao_vertical.pdf $\geq$ Acesso em: 20 de outubro de 2018.

BRASIL, Boletim Epidemiológico de Sifilis. Secretaria de Vigilância em Saúde - Ministério da Saúde. Disponível em: < http://www.aids.gov.br/pt-br/pub/2017/boletim-epidemiologico-de-sifilis2017 $\geq$ Acesso em: 20 de outubro de 2018.

BRASIL, Indicadores e dados básicos da sífilis nos municípios brasileiros. Departamento de DST, HIV e Hepatites Virais. Secretaria de Vigilância em Saúde. Disponível em <http://indicadoressifilis.aids.gov.br/> Acesso em: 20 de outubro de 2018.

BRASIL, Diretrizes para o Controle da Sífilis Congênita. Ministério da Saúde. Secretaria de Vigilância em Saúde. Programa Nacional de DST e AIDS. Brasília - DF 2006. Disponível em $<$ http://bvsms.saude.gov.br/bvs/publicacoes/manual_sifilis_bolso.pdf> Acesso em: 15 de outubro de 2018.

CHAVES, J. et al. Sífilis congênita: análise de um hospital do interior do estado do RS. Revista da Associação Médica do Rio Grande do Sul, v. 58, n. 3, p. 187-192, 2014.

DOMINGUES, R. M. S. M. et al. Prevalência de sífilis na gestação e testagem pré-natal: Estudo Nascer no Brasil. Revista de Saúde Pública, v. 48, n. 5, p. 766-774, 2014. 
DOMINGUES, R. M. S. M., LEAL, M. C. Incidência de sífilis congênita e fatores associados à transmissão vertical da sífilis: dados do estudo Nascer no Brasil. Caderno de Saúde Pública, Rio de Janeiro, 32(6):e00082415, 2016.

FRANÇA, I. S. X. et al. Fatores associados à notificação da sífilis congênita: um indicador de qualidade da assistência pré-natal. Revista da Rede de Enfermagem do Nordeste, v. 16, n. 3, p. 374-381, 2015.

FSVC, FUNDAÇÃO DE SAÚDE DE VITÓRIA DA CONQUISTA RECEBE VISITA DO MPF PREFEITURA MUNICIPAL DE VITÓRIA DA CONQUISTA. Disponível em: <http://www.pmvc.ba.gov.br/fundacao-de-saude-de-vitoria-da-conquista-recebe-visita-do-mpf/>. Acesso em: 20 de maio de 2018.

GUIMARÃES, T. A. et al. Sífilis em gestantes e sífilis congênita no Maranhão. Revista Arquivos de Ciências da Saúde, Rio de Janeiro, 25(2), p. 24-30, 2018.

LAFETÁ, K. R. G. et al. Sífilis materna e congênita, subnotificação e difícil controle. Revista Brasileira de Epidemiologia, v. 19, n. 1, p. 63-74, 2016.

MOREIRA, S. M. et al. Perfil dos casos notificados de sífilis congênita. Revista Cogitare Enfermagem, v. 22, n. 2, p. 2-10, 2017.

NONATO, K. F. A.; MELO, A. P. S.; GUIMARÃES, M. D. C. Sífilis na gestação e fatores associados a sífilis congênita em Belo Horizonte - MG, 2010-2013. Revista Epidemiologia e Serviços de Saúde, v. 24, n. 4 , p. 681-694, 2015.

SANTOS, S. G. et al. Aspectos epidemiológicos da sífilis congênita associados à escolaridade materna. Revista de Enfermagem, v. 10, n. 8, p. 2845-2852, 2016.

SILVA, T. Z. et al. Subnotificação de sífilis em gestantes, congênita e adquirida entre povos indígenas em Mato Grosso do Sul, 2011-2014. Revista Epidemiologia e Serviços de Saúde, v. 26, n. 3, p. 503$512,2017$.

SARACENI, V., VELLOZO, V., LEAL, M. C., et al. Estudo de confiabilidade do SINAN a partir das Campanhas para a Eliminação da Sífilis Congênita no Município do Rio de Janeiro. Revista Brasileira de Epidemiologia, v. 8, n.4, p. 419-24, 2005.

SILVA, G. A., DE OLIVEIRA, C. M. G. O registro das doenças de notificação compulsória: a participação dos profissionais da saúde e da comunidade. Revista de epidemiologia e controle de infecção. v. 4, n. 3. JUL/SET 2014.

REIS, J. G. et al. Diferenciais intraurbanos da sífilis congênita: análise preditiva por bairros do Município do Rio de Janeiro, Brasil. Cadernos de Saúde Pública, v. 34, n. 9, p. 1-13, 2018.

\section{Como citar este artigo (Formato ABNT):}

AZEVEDO, Débora Maria Santos; REIS, Rosilaine Barbosa Silva; TELES, Mauro Fernandes. Incidência e Caracterização dos Casos de Sífilis Congênita na Maternidade de um Hospital do Sudoeste Baiano . Id on Line Rev.Mult. Psic., 2019, vol.13, n.43, p. 387-397. ISSN: 1981-1179. 